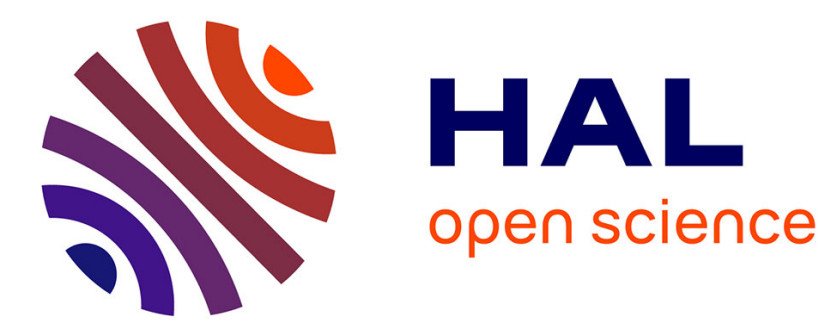

\title{
Les déterminants de la demande de protection : le cas français
}

Patrick Messerlin

\section{To cite this version:}

Patrick Messerlin. Les déterminants de la demande de protection: le cas français. Revue Economique, 1982, 33 (6), pp.1001-1023. 10.3406/reco.1982.408695 . hal-01009226

\section{HAL Id: hal-01009226 \\ https: / hal-sciencespo.archives-ouvertes.fr/hal-01009226}

Submitted on 17 Jun 2014

HAL is a multi-disciplinary open access archive for the deposit and dissemination of scientific research documents, whether they are published or not. The documents may come from teaching and research institutions in France or abroad, or from public or private research centers.
L'archive ouverte pluridisciplinaire HAL, est destinée au dépôt et à la diffusion de documents scientifiques de niveau recherche, publiés ou non, émanant des établissements d'enseignement et de recherche français ou étrangers, des laboratoires publics ou privés. 


\title{
Persée
}

http://www.persee.fr

\section{Les déterminants de la demande de protection : le cas français}

\author{
Patrick A. Messerlin \\ Revue économique, Année 1982, Volume 33, Numéro 6 \\ p. $1001-1023$
}

Voir l'article en ligne

\section{Avertissement}

L'éditeur du site «PERSEE » - le Ministère de la jeunesse, de l'éducation nationale et de la recherche, Direction de l'enseignement supérieur, Sous-direction des bibliothèques et de la documentation - détient la propriété intellectuelle et les droits d'exploitation. A ce titre il est titulaire des droits d'auteur et du droit sui generis du producteur de bases de données sur ce site conformément à la loi n`98-536 du 1 er juillet 1998 relative aux bases de données.

Les oeuvres reproduites sur le site «PERSEE » sont protégées par les dispositions générales du Code de la propriété intellectuelle.

Droits et devoirs des utilisateurs

Pour un usage strictement privé, la simple reproduction du contenu de ce site est libre.

Pour un usage scientifique ou pédagogique, à des fins de recherches, d'enseignement ou de communication excluant toute exploitation commerciale, la reproduction et la communication au public du contenu de ce site sont autorisées, sous réserve que celles-ci servent d'illustration, ne soient pas substantielles et ne soient pas expressément limitées (plans ou photographies). La mention Le Ministère de la jeunesse, de l'éducation nationale et de la recherche, Direction de l'enseignement supérieur, Sous-direction des bibliothèques et de la documentation sur chaque reproduction tirée du site est obligatoire ainsi que le nom de la revue et- lorsqu'ils sont indiqués - le nom de l'auteur et la référence du document reproduit.

Toute autre reproduction ou communication au public, intégrale ou substantielle du contenu de ce site, par quelque procédé que ce soit, de l'éditeur original de l'oeuvre, de l'auteur et de ses ayants droit.

La reproduction et l'exploitation des photographies et des plans, y compris à des fins commerciales, doivent être autorisés par l'éditeur du site, Le Ministère de la jeunesse, de l'éducation nationale et de la recherche, Direction de l'enseignement supérieur, Sous-direction des bibliothèques et de la documentation (voir http://www.sup.adc.education.fr/bib/ ). La source et les crédits devront toujours être mentionnés. 


\title{
LES DETERMINANTS \\ DE LA DEMANDE DE PROTECTION : LE CAS FRANÇAIS *
}

\author{
INTRODUCTION
}

Après avoir longtemps ignoré l'Etat et son rôle dans la vie économique, les économistes, notamment sous l'influence de Pigou et plus encore de Keynes, ont révéré cette institution, lui accordant une connaissance approfondie des événements passés, présents et futurs, ainsi que le comportement d'un despote «bienveillant». Cette vision de l'Etat, dominée par un «calcul économique» excessivement soucieux de professionnalisme, et apparue par trop ignorante des réalités politiques et économiques, et des interactions entre allocation de ressources et distribution des revenus. Depuis quelques années, la puissance des instruments d'analyse néo-classiques a été utilisée pour étudier l'institution étatique de façon plus profonde et réaliste. Cette institution apparaît alors davantage comme un assemblage hétéroclite de pouvoirs, arc-boutés sur des groupes ayant de forts intérêts spécifiques et organisés autour de politiciens ou de bureaucraties, que comme une construction harmonieusement dominée par la déesse Raison. Ce qui caractérise cependant cette nouvelle analyse économique de l'Etat, c'est le maintien du principe d'un comportement rationnel de chacun de ces groupes et pouvoirs, avec toutes les caractéristiques usuelles; mais, désormais, chacun de ceux-ci est essentiellement motivé par l'intérêt du groupe, et non plus par l'intérêt de l'ensemble de la collectivité nationale.

Appliquée au commerce international, et aux choix en matière de politique douanière et commerciale, cette nouvelle approche de l'institution étatique se révèle très fructueuse, notamment pour résoudre

* Je tiens à remercier ici S. T. Easton et $\mathrm{H}$. Schleicher pour leurs compentaires précieux à l'occasion de ce travail. 


\section{Revue économique}

cette étonnante contradiction surgie de l'histoire : pourquoi les forces protectionnistes sont-elles réapparues, par trois fois au moins, au cours des décennies 1890,1930 et 1980 , depuis le choix historique de la Grande-Bretagne en faveur du libre-échange, dans la première moitié du xrx siècle? Certains (Bairoch [1978]) avaient suggéré l'existence d'une corrélation entre un taux de croissance plus élevé et une protection renforcée. Mais cette hypothèse est des plus discutables, tant sur le plan théorique que dans les faits. (Messerlin [1980]) en sorte que la contradiction entre la résurgence de protectionnismes plus aigus et les bienfaits d'échanges plus libres demeure intacte tant que n'est pas pris en compte le comportement des institutions étatiques. Que des politiciens ou des fonctionnaires se fassent l'avocat de la protection s'explique on effet plus aisément si l'on veut bien considérer ces agents politico-économiques comme des décideurs rationnels, pour lesquels la situation protectionniste apporte plus d'avantages que d'inconvénients. Après tout, l'homme politique demeure l'avocat des intérêts de ses électeurs, et le fonctionnaire celui des intérêts de l'administration dont dépend sa carrière. Compte tenu des dispositions institutionnelles, l'un et l'autre utilisent leurs capacités à s'inter posex entre les mécanismes de marché impliqués par le libre-échange et les mécanismes politiques nés de l'existence des groupes de pression. Tôt ou tard, des politiciens ou fonctionnaires seront donc incités à «offrir de la protection » pour répondre à la «demande» exprimée par les groupes de pression.

Cette approche de la protection est parfaitement cohérente avec la théorie pure du commerce international : elle découle des mécanismes résumés par le théorème de Stolper-Samuelson. Elle a été progressivement élaborée dans une littérature, déjà abondante, dite de l'«économie politique du protectionnisme», qui a pris sa source dans les travaux précurseurs de Downs [1957] et d'Olson [1965] et qui comprend essentiellement les travaux de Krueger [1974], Breton [1974], Magee et Brock [1974], Pincus [1975], Caves [1976], Anderson [1979] et Baldwin [1982]. Cette approche a fait l'objet de tests nombreux concernant la plupart des pays indùstrialisés.

Ce papier a deux objectifs. D'une part, il offre des tests de cette approche dans le cas français, en cherchant, autant que possible, à utiliser des procédures d'estimation proches de celles utilisées pour les. autres pays, afin de suggérer quelques comparaisons intéressantes. D'autre part, il propose une explication à un phénomène, heureusement rare, mais significatif de l'entrée des pays dans une phase de protectionnisme aiguë : ce phénomène est celui de la «contagion 
protectionniste» au cours de laquelle un pays protège non plus seulement certaines de ses industries (au profit de celles-ci, et au détriment des autres), mais l'ensemble de ses activités éconorniques, dans un retour à l'autarcie qui condamne aussi bien les industries concurrencées par les importations que les industries exportatrices et qui amène inéluctablement une baisse du bien-être collectif.

\section{LES DETERMINANTS \\ DE LA PROTECTION FRANGAISE : ETUDES EMPIRIQUES}

La première approche que nous utiliserons pour tester les déterminants de la demande de protection a été définie par E. J. Ray [1981]. Celui-ci insiste sur le fait que le comportement traditionnel de maximisation des profits s'applique au cas des restrictions douanières et non douanières, lesquelles deviennent une variable d'action, déterminant les profits dans un environnement politique donné. Dès lors, le profit que chaque industrie retire des restrictions aux importations dépend de deux grands groupes de variables : d'une part, les caractéristiques économiques liées aux avantages ou aux désavantages comparatifs de l'industrie considérée; d'autre part, les «facteurs politiques» tels que retenus par la littérature consacrée à l'économie politique du protectionnisme. L'équation à tester est donc du type général :

$$
t=t(\mathrm{AC}, \mathrm{FP})
$$

où $t$ représente le niveau de protection (droits de douane ou barrières non tarifaires), AC les variables représentant les avantages comparatifs et FP celles représentant les facteurs politiques.

Le signe espéré pour les variables représentant les avantages comparatifs découle de l'argument général suivant : plus une industrie est internationalement compétitive, c'est-à-dire plus elle jouit d'un avantage comparatif au sens du critère retenu, plus faibles sont les profits qu'elle peut espérer retirer des restrictions à l'importation, si l'on exclue la possibilité, pour cette industrie, dobtenir, par cette protection, des rentes sur le marché français, en imposant une politique 


\section{Revue économique}

des prix de «dumping ».1. Comme il n'existe pas d'indicateur synthétique des avantages comparatifs, Ray adopte une approche «éclectique » faisant usage des principaux indicateurs disponibles pour mesurer les avantages comparatifs, soit le coefficient d'intensité factorielle KLQ, l'intensité en travail LSH, l'intensité en travail qualifié SIQ, et l'intensité en recherche-développement RDQ ${ }^{2}$. Selon les rares études disponibles pour la France, les exportations françaises seraient intensives en travail et plutôt intensives en travail qualifié ${ }^{3}$. Ces résultats nous font espérer les signes suivants pour l'équation (1) développée dans ses arguments d'avantages comparatifs.:

$$
t=t\left(\mathrm{KLQ}_{+}, \mathrm{LSH}, \mathrm{SIQ}, \underset{?-}{\mathrm{RDQ}}, \mathrm{FP}\right)
$$

Trois remarques doivent accompagner ces signes espérés. En premier lieu, les indicateurs retenus l'ont été au titre d'approximations des avantages comparatifs. Mais ils ont aussi une signification en termes du modèle à facteurs spécifiques. Si le travail est globalement un facteur mobile, aucun signe a priori ne peut être affecté à l'intensité en travail LSH, puisque le facteur mobile est relativement "neutre » à l'égard de la politique commerciale suivie. Par contre, des signes espérés peuvent être affectés à ces facteurs spécifiques que peuvent être, par exemple, le capital ou le travail qualifié, selon qu'ils sont liés aux industries des importables ou à celles des exportables. La seconde remarque concerne deux variables que Ray introduit en plus des mesures des avantages comparatifs : augmenter la protection, c'est-àdire les prix domestiques, est d'autant plus profitable qu'est faible l'élasticité de la demande domestique et forte celle de l'offre étrangère. Considérer cette relation entre protection d'une part et demande et offre d'autre part se heurte, malheureusement, à la difficulté de disposer d'estimations convenables des élasticités en jeu, notamment pour la demande domestique 4 . Enfin la troisième remarque concerne l'argu-

1. Au niveau d'agrégation de l'échantillon utilisé, de telles situations ne paraissent guère observables, même dans les industries de l'énergie, de l'automobile ou de l'armement.

2. La définition de ces variables figure dans l'annexe.

3. Cf. Prouteau [1967], Vellas [1978] qui n'envisagent cependant pas de façon autonome l'intensité en recherche-développement, ce qui ne nous permet pas de donner un signe a priori à cette variable.

4. La variable proxy utilisée par Ray, à savoir le critère habituellement utilisé dans les tests des avantages comparatifs de la différenciation des produits, n'est pas satisfaisante. Comme le notent Anderson et Baldwin [1981], si la courbe d'offre étrangère est infiniment élastique, comme nous le supposons en traitant la France de "petit pays ", le prix domestique croît à seule proportion du droit de douane et l'élasticité de la demande ne joue un rôle que sí toute importation cesse. 
ment classique du «droit de douane optimal », c'est-à-dire l'existence de situations de monopsone/monopole sur les marchés étrangers pour des industries françaises : comme Ray, nous considérons de telles situations comme suffisamment rares et limitées pour être négligées dans cette étude. Quant au dernier argument classique de la théorie de la protection, celui de «l'industrie dans l'enfance», Ray n'en souffle mot : Johnson [1970] a montré que cet argument n'est recevable que dans des cas fort limités d'externalités et qu'il se ramène, le plus souvent, à une argumentation commode, mais inexacte, à la disposition des groupes de pression.

Il convient maintenant de développer les facteurs politiques de l'équation à tester. Avant de comparer nos résultats avec ceux de Ray, nous reprendrons dans un premier temps les trois facteurs retenus par Ray. Le premier est le niveau de concentration au sein de chaque industrie. De l'avis de la plupart des observateurs de la vie politique et économique entourant les choix de politique commerciale, le niveau de concentration est un facteur important. Malheureusement, cette constatation ne permet pas de savoir dans quel sens joue ce facteur. Est-ce en faveur de la protection, grâce au pouvoir de négociation acquis par les firmes concentrées, grâce au fait qu'elles sont particulièrement sensibles aux facteurs spécifiques, ou encore qu'elles savent mieux organiser (à un coût moins élevé) de puissants lobbies ? Ou, au contraire, est-ce en faveux du libre-échange, grâce aux économies d'échelle que ces grandes firmes sont supposées (hâtivement) mieux développer que d'autres, à cause des intérêts importants qu'elles ont à l'étranger et qui les rendent sensibles aux menaces étrangères de représailles, ou encore à cause de la faiblesse politique qui accompagne, de façon quelque peu paradoxale mais réelle, leur puissance économique? Il est clair, donc, qu'il ne peut y avoir de signe a priori évident pour cette variable de concentration CNQ. Cependant, compte tenu de l'année utilisée pour notre test [1974], nous pouvons nous risquer à suggérer un signe espéré, sur la base de considérations « ad hoc »: l'environnement national et international suggère plutôt un signe négatif, les grandes firmes internationales situées en France étant logiquement en faveur du libre-échange, et les grandes firmes nationales entamant, à cette époque, un effort d'ouverture certain et prenant conscience des interdépendances internationales. Le second facteur politique est celui de l'asymétrie de l'information disponible par les producteurs et consommateurs à propos de la politique commerciale : de trop fréquentes variations de celle-ci en faveur d'une industrie ne peuvent qu'attirer l'attention des consommateurs sur l'inefficience de 


\section{Revue économique}

cette industrie, et le coût des restrictions aux importations en résultant pour leur pouvoir d'achat. Dès lors, les industries peuvent rechercher les formes de protection les moins voyantes pour échapper à la sanction des consommateurs. Ces formes de protection discrètes varient en raison de la situation des marchés en jeu : Ray' suggère que les restrictions douanières seront préférées par les producteurs pour protéger les narchés dont la dẹmande domestique et/ou l'offre étrangère décroissent, et que les restrictions quantitatives seront préférées dans le cas inverse. Les mesures approximatives satisfaisantes de ces deux types de marché sont difficiles à trouver; aussi Ray se contente-t-il de reprendre les mesures habituelles de l'évolution de la consommation apparente, DCO, et celle de la pénétration du marché intérieur, DIM 5 ? Enfin, le dernier facteur politique est celui de la perte globale en bienêtre découlant de la protection : si les autorités publiques sont attentives en la matière, elles doivent accorder un soin tout particulier à la hausse des coûts entraînée par la fermeture des frontières. Une mesure de cette relation des coûts avec le niveau de protection peut être trouvée avec les économies d'échelle (SAQ) dont le signe espéré est à l'évidence négatif. La relation à tester est maintenant totalement développée :

$$
\left.t=t \underset{+}{(\mathrm{KLQ}}, \underset{-}{\mathrm{LSH}}, \underset{-}{\mathrm{SIQ}}, \underset{-?}{\mathrm{RDQ}}, \underset{-?}{\mathrm{CNQ}}, \mathrm{SAQ}_{-}, \underset{+}{\mathrm{DOC}}, \underset{+}{\mathrm{DIM}}\right)
$$

Les résultats, portant sur les 23 premières branches de la nomenclature NAP 40 (ou parfois sur 22 branches, l'agriculture (NAP 01) étant exclue), figurent dans le tableau 1 , colonnes $\mathrm{A}$ et $\mathrm{B}$. Ces deux colonnes ont pour variable dépendante la moyenne simple des droits de douane nominaux. En effet, aucune des spécifications de l'équation (2) n'est significative si l'on considère les droits de douane nominaux pondérés par les importations (droits de douane nominaux moyens), ceci tenant vraisemblablement au petit nombre d'observations, et au fait que les droits de douane agrégés en NAP 40 ne retransmettent qu'imparfaitement la dispersion des droits selon les industries, et la plage des variations possibles pour ceux-ci ${ }^{6}$. Les résultats contenus dans les colonnes $\mathrm{A}$ et B sont intéressants à un double titre. D'une part, la seule variable

5. Il est évident que ces deux variables sont des proxys médiocres puisqu'elles sont le reflet d'à la fois l'offre et la demande. Il demeure cependant que leur impact " politique " est substantiel et que, pour cette raison, elles méritent considération.

6. A titre d'exemple, les droits de douane nominaux moyens varient de 0 à $12 \%$ dans la NAP 40, et de 0 à $32 \%$ dans la NAP 100 , pour la même année 1974 et le même champ d'observation. 
significative est l'intensité en travail qualifié. Cette variable est significative dans toutes les autres spécifications de l'équation qui ont un F suffisant ; les autres variables liées aux avantages comparatifs (KLQ, LSH et RDQ) ne le sont jamais. Enfin, le signe de SIQ est toujours négatif, ce qui est le signe espéré. D’autre part, les économies d'échelle, qui sont d'ailleurs précédées d'un signe positif, contraire à celui espéré, n'apparaissent pas dans le cas français comme une variable significative. Une interprétation de ce point, liée à la politique industrielle des autorités publiques françaises, sera proposée une fois introduites de nouvelles variables explicatives.

Enfin, il convient de modifier le canevas de l'analyse de Ray pour prendre en compte des facteurs plus propres à l'environnement économique et politique français. Trois modifications sont envisagées. Les deux premières portent sur les variables indépendantes. Tout d'abord, B. Balassa [1981] a suggéré une hypothèse intéressante selon laquelle le commerce intrabranche donnerait lieu à moins de pressions protectionnistes que le commerce interbranche. La raison en serait que les firmes seraient plus aptes à s'adapter en cas de pénétration intrabranche car elles se heurteraient à moins de rigidités législatives, psychologiques, technologiques et économiques auprès des facteurs de production qu'elles emploient. Il serait, en général, plus facile pour les firmes de quitter certains créneaux pour sé spécialiser dans d'autres en utilisant le même personnel et/ou le même stock de capital.

A côté des variables économiques d'avantages comparatifs, nous ajoutons donc un indicateur du commerce intrabranche CIQ, avec pour signe espéré un signe négatif ${ }^{7}$. L'autre variable indépendante à considérer concerne les facteurs politiques. Les industries françaises, tout le laisse à penser, ont pris thabitude de vivre dans un environnement très réglementé : en matière de politique commerciale, ceci veut dire qu'elles sont habituées à un minimum de barrières non tarifaires, aux formes multiples, et qu'elles considèrent de telles barrières comme allant de soi. Aussi, apparaît-il justifié d'introduire, comme variable indépendante, une variable dummy $(0,1)$ représentant $l e$ « roc» de ces barrières non tarifaires. La demande de protection douanière, à un moment donné, doit être alors comprise comme le supplément de protection que chaque industrie désire en plus de cette protection minimale non tarifaire : plus élevée est cette protection «naturelle», plus faible est la protection douanière, voyante donc, demandée en

7. Dans une certaine mesure ; cet indicateur "CIQ " est le reflet des économies d'échelles intrabranches qui seraient une des raisons essentielles du stuccès de la CEE. 
supplément. Enfin, la troisième modification à apporter au test initial de Ray est de considérer, pour variable dépendante, non plus les droits de douane nominaux mais soit les droits de douane effectifs, soit les barrières non tarifaires ${ }^{8}$. L'équation à tester devient :

$$
\mathbf{P}=\mathbf{P}(\mathrm{KLQ}, \mathrm{LSH}, \mathrm{SIQ}, \mathrm{RDQ}, \mathrm{CIQ}, \mathrm{CNQ}, \mathrm{SAQ}, \mathrm{DOO}, \mathrm{DIM}, \mathrm{NBQ})
$$

où $\mathrm{P}$ représente le niveau de protection tarifaire ou non tarifaire. Les résultats figurent dans le tableau I, colonnes C, D et E. Quatre constatations importantes peuvent être faites en ce qui concerne les spécifications avec droits de douane effectifs. Tout d'abord, on note que l'intensité en travail qualifié a perdu toute significativité (et il en est de même dans toutes les autres spécifications qui ont pu être faites), au contraire de la variable concentration. Ceci découle sans doute de ce que ces deux variables sont colinéaires, ainsi que le sont, dans une moindre mesure, concentration et coefficient d'intensité factorielle. Néanmoins, lịnterprétation suivante peut être suggérée : la politique industrielle menée par les autorités publiques a conduit à la création de «champions nationaux»; cette politique a abouti à un effort de concentration, accompagné d'un accroissement de l'intensité en travail qualifié 9 , et a concordé avec l'ouverture des frontières. En second lieu, la variation de la consommation apparente joue un rôle significatif, dans le sens espéré.

Troisièmement, le commerce intrabranche, lui aussi significatif, est par contre affecté d'un signe opposé à celui espéré. On peut se demander si ceci ne reflète pas une réelle difficulté à se réorganiser au sein d'une même branche, contrairement à l'hypothèse de Balassa :- à cause d'évolutions technologiques différentes, deux produits différents d'une même branche peuvent requérir davantage d'ajustements que deux produits de branches différentes ${ }^{10}$. Enfin, la variable des barrières non

8. Les droits de douane effectifs ont été calculés sur la base des seuls droits nominaux, à l'exclusion de toutes subventions ou autres aides inclirectes des autorités publiques : il s'agit donc d'une estimation "partielle ". L'évaluation des bar" rières non tarifaires est évidemment chose sujette à de nombreuses difficultés : nous avons adopté une attitude simple consistant à considérer les "Hearings" du Sénat des Etats-Unis comme constituant un rapport d'experts, d'autant plus impartial qu'il ne traite pas que du cas français.

9. On peut se demander, de façon moins innocente et peut-être plus pertinente, si la plus forte intensité en travail qualifé n'est pas une retombée de la création des champions nationaux et des situations monopolistiques que ceux-ci se sont vus accordées.

10. De plus, le niveau d'agrégation, tant en matière de branches gue de pays, peut avoir biaisé les résultats. 
tarifaires, affectée du signe espéré, n'atteint pas, de peu, le seuil de significativité.

Les résultats concernant les barrières non tarifaires figurent dans le tableau 1, colonnes $F$ à $J$. Dans les deux premières colonnes, les barrières sont calculées en valeur absolue, représentant la quantité d'importations soumises à des restrictions non douanières; dans les deux dernières, elles sont calculées en pourcentage de la quantité totale d'importations du monde (CEE exclue). Dans les colonnes F et G, deux nouvelles variables significatives apparaissent : l'intensité en travail et celle en recherche-développement. Cette dernière, accompagnée d'un signe positif, confirme qu'il existe un renforcement «automatique » de la protection des produits sophistiqués, dî̀ aux normes technologiques, à la compatibilité des matériels, etc. ; elle suggère encore que tout ce qui touche à la recherche-développement est volontairement protégé, quand il l'est, par des barrières occultes ou indirectes, plutôt que par des droits de douane. Dans les colonnes I et J, la variable des économies d'échelle apparaît, pour la première fois, significative. Son signe positif mériterait une réflexion approfondie. On peut simplement avancer ici que, si l'on associe économies d'échelle et différenciation des produits ${ }^{11}$, les droits de douane n'apparaissent pas comme le moyen optimal de protection, car il risque de déclencher des représailles portant atteinte aux exportations et donc au jeu des économies d'échelle, et car il est mal adapté, les nomenclatures douanières, même détaillées, ne pouvant généralement saisir les finesses de la différenciation; aussi, les entreprises se tournent-elles vers les barrières non tarifaires qui peuvent être taillées sur mesure et limiter les risques de représailles.

La seconde approche utilisée ici est celle qui a été adoptée par Waelbroeck et Baldwin (en abrégé, WB) pour un ensemble de pays industrialisé ${ }^{12}$. Cette approche se distingue de celle de Ray dans la mesure où elle insiste davantage sur les marchés politiques qui naissent de l'offre et de la demande de protection, et où, en conséquence, elle laisse dans l'ombre les indicateurs liés aux avantages comparatifs ${ }^{13}$. L'approche WB insiste tout particulièrement sur les différences interindustrielles dans la demande de protection au sein d'un pays. Ces différences naissent du jeu de trois types de forces économiques.

11. Conformément à une tendance récente dans la littérature, en matière de théorie pure du commerce international, cf. Krugman [1978].

12. Cette approche a été résumée dans Anderson et Baldwin [1981], dont nous faisons usage pour décrire l'équation (3). B. Bobe [1980] dans un papier non publié, avait commencé à appliquer cette méthode au cas français.

13. Et quand elle reprend une variable utilisée comme indicateur des avantages comparatifs dans l'approche de Ray, elle lui donne une autre signification : ceci est le cas de l'intensité en travail, LSH. 


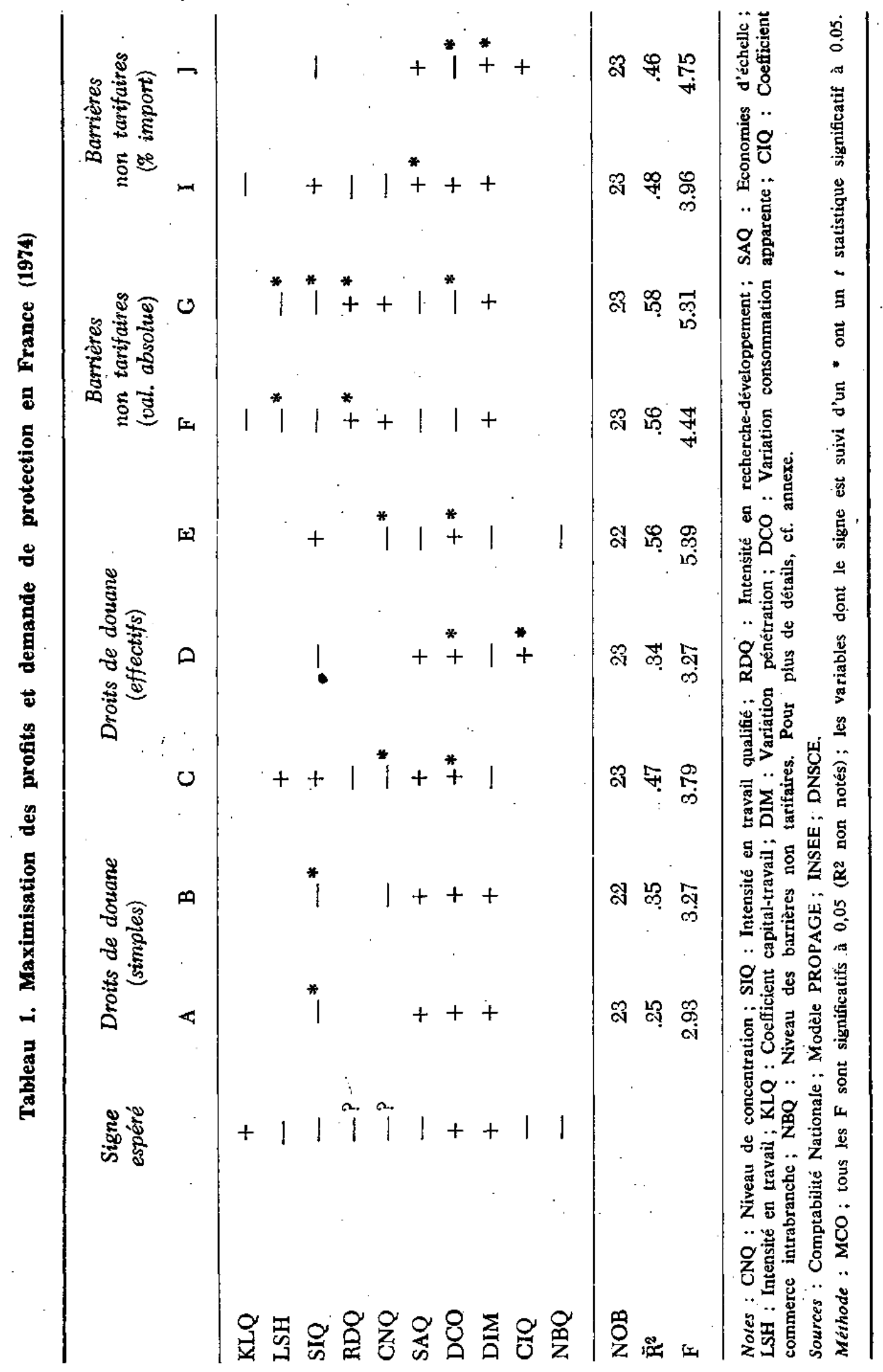


En premier lieu, la capacité d'une industrie à faire pression, et donc à obtenir de la protection, dépend de sa structure en termes de firmes et de marchés : mais moins il y a des firmes dans l'industrie (NOF), plus celle-ci est concentrée (CNQ), plus il est facile, peu coûteux, de constituer un groupe de pression, de le gérer, et d'obtenir des gains en proportion avec les coûts car on ne se heurte pas au problème du "passager clandetin» (free rider). Néanmoins, le signe espéré pour CNQ est, encore une fois, contrebalancé par d'autres forces que nous avons évoquées lors de l'approche de Ray, et se révèle plutôt incertain.

En second lieu, la demande de protection est fonction des motivations des principaux facteurs de production. Si le capital est un facteur plus spécifique que le travail (a une offre plus inélastique que ne l'a l'offre de travail), la protection accroîtra ses revenus d'autant plus que l'industrie sera intensive en travail (LSH). De plus, les gains, pour les propriétaires d'une firme, résultant d'un accroissement des prix des produits fabriqués grâce à la protection, seront d'autant plus grands que sera faible la part de la valeur ajoutée dans la production (VSH). Quant aux travailleurs, ils se joindront aux capitalistes dans la demande de protection avec d'autant plus d'empressement que l'évolution de l'emploi est défavorable (DCO) et que le niveau de qualification, illustré par le salaire moyen (AWQ), est faible, ces deux caractéristiques retranscrivant les difficultés futures d'emploi auxquelles peuvent s'attendre les travailleurs licenciés par l'industrie.

Enfin, cette capacité à créer des groupes de pression et à demander de la protection dépend de l'environnement général de la branche. Si celle-ci est exportatrice (ESQ), elle doit tenir compte des représailles éventuelles sur ses exportations, à la suite d'une protection accrue contre les biens importés concurrençant sa production, et elle doit donc réduire sa demande de protection en conséquence. Si elle produit des biens dont la valeur ajoutée par employé est forte (VLQ), il est vraisemblable qu'elle pourra se contenter d'un niveau de protection plus faible que dans le cas contraire qui la verrait exposée à la concurrence des pays industrialisés et en voie de développement. A ces considérations, il faut ajouter le fait qu'un groupe de pression ne naît pas spontanément, ou n'agit pas immédiatement, mais à la suite de revers et de "coûts» successifs ressentis par l'industrie : celle-ci doit done se sentir déclinarite, soit dans ses capacités productives (DPR), soit dans sa domination du marché intérieur. (ISQ). Enfin, une industrie, relativement importante par rapport aux autres, en termes d'effectifs employés par exemple (LOQ), et/ou mal protégée 
par ces barrières naturelles que sont les coûts de transports (DTC); se sentira en droit de demander davantage de protection.

L'équation générale à tester est donc du type :

$$
\mathrm{P}=\mathrm{P}\left(\underset{-}{\mathrm{NOF}}, \underset{++}{\mathrm{CNQ}}, \mathrm{LSH}, \mathrm{VSH}, \mathrm{DLO}, \mathrm{ANQ}_{-}, \mathrm{ESQ}, \mathrm{VLQ}_{-}, \mathrm{DPR}_{+}, \underset{+}{\mathrm{ISQ}}, \underset{+}{\mathrm{LOQ}}, \mathrm{PTC}\right.
$$

dans laquelle $\mathbf{P}$ représente le niveau de protection, tarifaire et/ou non tarifaire. Les résultats figurent dans le tableau 2. Seuls parmi les cinq variables endogènes retenues, les droits de douane effectifs et les barrières non tarifaires (en valeur absolue) ont abouti à des estimations significatives. En ce qui concerne les droits de douane effectifs (colonnes $\mathrm{A}$ à $\mathrm{C}$ ), nous retrouvons, comme variable significative, le niveau de concentration, avec un signe contraire à celui espéré dans le cas général des pays industrialisés. Les deux autres variables significatives (pénétration des importations et rapport d'exportations) apparaissent aussi avec des signes opposés à ceux espérés, ce qui semble pouvoir être expliqué par le fait que ces deux variables sont exprimées ici en termes de niveau (en sorte qu'elles ne saisissent pas les modifications structurelles au sein de branches aussi agrégées que celles en NAP 40), et non pas en termes de variations, comme dans l'approche de Ray où les signes espérés avaient été obtenus. Enfin, les variables ajoutées à l'équation de.WB, bien qu’avec le signe espéré, ne sont pas significatives.

Plus riche en interprétation est l'estimation en termes des barrières non tarifaires. Le rôle des facteurs de production apparaît clairement, et dans le sens espéré, d'une part, avec l'intensité en travail (reflet de la spécificité du capital) et, d'autre part, avec la variation du nombre des employés dans l'industrie. Enfin, les trois autres variables significatives suggèrent les trois enseignements suivants. En premier lieu, une augmentation de la production au cours du temps est liée à une augmentation des barrières non tarifaires (DPR) : ceci paraît bien recouvrir le fait que certaines industries n'ont dû cette évolution favorable de leur niveau de production qu'à la protection passée, et qu'elles ne peuvent espérer maintenir cette évolution qu'en augmentant leurs pressions protectionnistes. En secònd lieu, limportance des branches en terme de travailleurs est négativement reliée aux barrières non tarifaires : ceci pourrait s'expliquer par l'aspect encore relativement marginal que pouvaient avoir les barrières non tarifaires au début des années 1970. Enfin, le nombre de firmes est positivement corrélé avec ces barrières : dans l'environnement «expansionniste» de l'année 1974, les entreprises croyaient largement en un élargissement des 
marchés; ce glissement continu espéré, vers la droite, de la demande pour la plupart des produits, s'il est totalement satisfait par des importations en cas de droits de douane, l'est, partiellement au moins, par la production domestique en cas de quota, cette production domestique étant le fait des unités de production marginales, lesquelles peuvent ètre mieux saisies par le nombre de firmes lorsque celui-ci est important.

Tableau 2. Protection et marchés politiques en France (1974)

\begin{tabular}{|c|c|c|c|c|c|}
\hline & \multirow[t]{2}{*}{$\begin{array}{l}\text { Signe } \\
\text { espéré }\end{array}$} & \multicolumn{3}{|c|}{ Droits de dotane effectifs } & \multirow{2}{*}{$\begin{array}{c}\text { Barrières } \\
\text { non tarifaires } \\
\text { (cal. absolue) } \\
\text { D }\end{array}$} \\
\hline & & $A$ & B & C & \\
\hline NOF & - & - & - & - & $+*$ \\
\hline CNQ & $?+$ & - $^{*}$ & -* & —* & + \\
\hline LSH. & + & - & - & + & $+\cdot$ \\
\hline VSH & 一 & - & - & + & + \\
\hline DLO & - & + & + & + & -* \\
\hline AWQ & - & + & + & + & + \\
\hline ESQ & - & $+*$ & $+*$ & $+*$ & - \\
\hline VLQ & - & & & & - \\
\hline DPR & - & + & + & + & $+*$ \\
\hline ISQ & + & - * & —* & - & + \\
\hline L.OQ & + & - & - & - & $-*$ \\
\hline DTC & $?$ & + & + & - & - \\
\hline CIQ & $\omega$ & & - & & \\
\hline NBQ & - & & & - & \\
\hline NOB & & 23 & 23 & 23 & 22 \\
\hline$\overline{\mathrm{R}}^{2}$ & & .65 & .61 & .65 & .55 \\
\hline $\mathrm{F}$ & & 4.37 & 3.63 & 4.10 & 3.40 \\
\hline
\end{tabular}

Not's : NOF : Nombre de firmes; CNQ : Niveau de concentration ; LSH : Intensité en travail ; VSH : Valeur ajoutce dans production; DLO : Variation de l'emploi; AWQ : Niveau de qualification : ESQ : Exportations sur production; VLQ : valeur ajoutée par employé ; DPR : Variation dans la production: ISQ : Pénétration des importations ; LOQ : Effectifs employés; DTC : Variation coûts de transport: CIQ : Coefficient eommerce intrabranche; NBQ : Niveau des barrières non tarifaires.

Sources : Comptabilité Nationale; INSEE : DNSCE.

Méthode : $\mathrm{MCO}$; tous les F sont significutifs à 0.05 ( $\mathbf{R}^{2}$ non notés); les variables dont le signe est suivi d'un * ont un t statistique significatif à 0,05 . 


\section{Revue économique}

Pour conclure, cette seconde approche confirme la première. Elle rappelle la sensibilité bien connue de la protection française aux flux d'échanges, notamment pénétration des marchés intérieurs et extraversion des industries. Mais elle fait apparaître aussi certains traits moins connus : le rôle ambigu de la concentration, rôle favorable sur les barrières non tarifaires moins voyantes; le rôle du facteur travail, très sensible à l'évolution de l'emploi, et aussi apte à obtenir des concessions de la part des autorités publiques hors de proportion par rapport à l'importance des effectifs employés; le rôle du facteur capital, enfin, sachant jouer de sa «spécificité 》 pour accroître ses rentes obtenues sur le marché français, ou poussant à maintenir en vie des firmes domestiques marginales.

\section{LA CONTAGION \\ DES MESURES PROTECTIONNISTES}

La politique commerciale des pays est parfois sujette à un phénomène remarquable qui est celui de la «contagion» des mesures protectionnistes : non seulement les industries fortement concurrencées par les importations demandent et obtiennent un relèvement de leur niveau de protection, mais aussi les industries largement exportatrices nettes cherchent à s'abriter derrière des barrières douanières ou non tarifaires. Une telle évolution a pu être constatée dans de nombreux pays, au cours des années 1930; au cours des années 1950 et 1960, elle a été une constante de la politique commerciale des pays en voie de développement; enfin, elle semble réapparaître de nos jours, au moins sous forme de souhaits et de désirs des groupes de pression, dans les pays industrialisés dont le nôtre.

Ce phénomène de contagion de la protection est contradictoire avec le concept même de protection, ce qui en fait un problème intéressant d'analyse économique. En effet, l'objet de toute protection est d'augmenter le prix relatif des biens d'une industrie par rapport aux prix des autres industries. Augmenter le prix de toutes les industries, ce à quoi aboutit la contagion des mesures protectionnistes, tend à annuler cet effet de prix relatifs qui constitue le cœur de la protection. Il est même possible que cette dispersion des mesures protectionnistes rapproche le niveau des prix relatifs domestiques du niveau des prix relatifs internationaux si les mesures de protection par les 
industries exportatrices nettes, que nous appellerons industries des exportables, sont davantage renforcées que les mesures dont bénéficient les industries fortement concurrencées par les importations, que nous appellerons industries des importables 14 .

Cet aspect paradoxal de la dispersion des mesures protectionnistes n'a guère été remarqué, parce que, jusqu'à présent, l'explication de ce phénomène de dispersion était recherchée dans l'existence de processus internationaux de représailles. Sans doute, cette explication n'estelle pas entièrement négligeable. Mais il est possible de trouver des exemples de dispersion des mesures protectionnistes sans constater l'existence de processus de représailles ${ }^{15}$.

Aussi, à ce phénomène de contagion de la protection, il convient de rechercher des raisons internes à l'économie considérée plutôt que des raisons extérieures. L'approche analytique rappelée dans l'introduction suggère une explication qui permet de comprendre le paradoxe de cette contagion de la protection : celle-ci apparaît comme la conséquence d'un jeu non coopératif entre partisans de la protection et ceux du libre-échange. Dans ce jeu non coopératif, du type du dilemme du prisonnier, les décideurs publics (politiciens, ou fonctionnaires, ou toute combinaison des deux) jouent un rôle essentiel à un double titre. D'une part, chaque décideur public épouse la cause du groupe de pression avec lequel il est lié : le politicien est un élu de la circonscription électorale dans laquelle l'industrie considérée est bien représentée en nombre de votes; le fonctionnaire appartient à l'organisme de tutelle de l'industrie examinée. D'autre part, le fait que le décideur public coopère parfaitement avec une industrie donnée renforce la vraisemblance d'une information imparfaite, laquelle est une condition nécessaire à l'existence d'un jeu non coopératif du type du dilemme du prisonnier.

Comme il est bien connu, le résultat d'un tel jeu non coopératif est l'existence de décisions collectives irrationnelles, protection accrue et/ou distorsions internes renforcées, alors que la rationalité économique demeure vérifiée au niveau « individuel » des joueurs, c'est-à-dire au niveau des industries et des décideurs publics. Le mécanisme de

14. Cette définition des industries des importables et des exportables nous permettront d'introduire le commerce intrabranche qui sera nécessaire pour comprendre ultérieurement l'attitude de l'industrie des exportables.

15. Dans le cas français, le mécanisme des représailles peut avoir joué pour les années 1930-1936. Mais ceci apparaît beaucoup plus douteux pour la fin du xix siècle, période au cours de laçuelle, si la France a connu des guerres tarifaires, elle a continué à bénéficier de la clause de la nation la plus favorisée avec ses principaux partenaires européens. 


\section{Revue économique}

ce jeu non coopératif menant à la contagion des mesures protectionnistes sera montré, dans un premier temps, avec le cas le plus simple, où il n'existe que deux industries, celle des importables et celle des exportables. Puis, dans un second temps, il sera étendu au cas d'une économie dotée d'une troisième industrie, celle des biens non échangeables.

\section{Le cas à deux industries}

Considérons, une «petite» économie, faisant face donc à des termes de l'échange internationaux fixes, dans laquelle chacune des industries emploie des facteurs de production spécifiques, que nous appellerons capital 16. Si l'industrie des importables I bénéficie de mesures protectionnistes, alors l'effet d'amplification (Jones, [1971]) montre que le capital de l'industrie $\mathrm{E}$ des exportables verra son taux de rendement réel décroître. Pour retrouver son pouvoir d'achat, ce capital spécifique à l'industrie $\mathrm{E}$ peut se faire, tout d'abord, l'avocat d'une politique de libre-échange : celle-ci lui assurera ine évolution favorable des termes de l'échange domestique. Mais, inévitablement, une telle politique de libéralisation des échanges lèsera les intérêts du capital spécifique de l'industrie I, et, par voie de conséquence, et ceci est important, les intérêts des décideurs publics coopérant avec cette industrie I. Pour contourner la double opposition de lindustrie I et des décideurs publics qui lui sont associés, l'industrie $\mathrm{E}$ doit chercher à leur offrir des compensations. L'acceptation ou le rejet de ces compensations par l'industrie I dépend de l'environnement économique national, et aussi des marchandages possibles entre les décideurs publics champions des deux industries.

Lorsque les compensations sont jugées insuffisantes par l'industrie I, l'industrie $\mathbf{E}$ peut recourir à la politique alternative consistant à obtenir la modification désirée des termes de l'échange domestiques grâce à des mesures protectionnistes déclenchant un effet d'amplification, mais cette

16. Pour une analyse du modèle à facteurs spécificues, cf. Caves et Jones [1981], chap. vr, p. 95-112. Rappelons que si $w$ est le salaire du travail, facteur mobile $r_{\mathrm{I}}$, le taux de rendement du capital spécifique à l'industrie $I, r_{E}$ le taux correspondant pour le capital spécifique à l'industrie $E$, si $P_{I}$ et $P_{E}$ sont les prix des biens des industries $I$ et $E$, et si " ${ }^{n}$ désigne la variation en pourcentage de la variable considérée, alors l'effet d'amplification s'écrit :

$$
\hat{r}_{I}>\hat{P}_{I}>\hat{x}>\hat{P}_{E}>\hat{r}_{E}
$$


fois-ci dans le sens souhaité par le capital spécifique à l'industrie $\mathbf{E}$. Il est clair, cependant, que la contagion des mesures protectionnistes découlant de cette nouvelle stratégie de l'industrie $\mathrm{E}$ exigera l'emploi de davantage d'hommes et de moyens dans cette activité de recherche de rentes qu'est la protection d'une industrie tant au niveau des groupes de pression dans leur demande de protection que des décideurs publics dans leur offre de protection.

Ce jeu, non coopératif, peut être illustré à l'aide de la matrice des gains en termes d'utilité du tableau 3. Chaque joueur, qui est une combinaison stable d'une industrie et des décideurs publics coopérant avec cette industrie, a seulement deux stratégies à sa disposition : être en faveur du libre-échange (L) ou de la protection (P). Si les deux joueurs $\mathrm{I}$ et $\mathrm{E}$ jouent la stratégie du libre-échange, les gains nets de l'échange (c'est-à-dire les gains dont ont été déduits les coûts d'ajustement économiques nécessités par louverture des frontières) doivent être suffisants pour assurer aux joueurs des gains d'utilité, soit, par exemple $(+2,+2)$. Si maintenant les deux joueurs I et $\mathbf{E}$ choisissent un renforcement de la protection de l'économie, les inefficiences accrues du fait de la mauvaise allocation des ressources, les distorsions introduites dans le fonctionnement de l'économie, les coûts impliqués par la fermeture des frontières (car les coûts d'ajustement n'existent pas seulement daris le cas d'une libéralisation des échanges mais apparaissent aussi dans le cas de restriction des échanges) entraîneront une baisse de l'utilité de chacun des deux joueurs, que nous supposerons égale à $(-2,-2)$. Restent enfin les deux stratégies où l'un des joueurs pratique la stratégie protectionniste en sa faveur, et donc gagne $(+4)$ grâce à l'effet d'amplification, tandis que l'autre, acceptant le libreéchange, perd (-3). Si l'information est imparfaite, les conditions sont alors réunies pour que le pays choisisse la pire solution, c'est-à-dire la contagion des mesures protectionnistes dans l'ensemble de l'économie.

Tableau 3. Contagion protectionniste avec deux industries

\begin{tabular}{|l|r|r|}
\hline & \multicolumn{2}{|c|}{ Industrie $\mathrm{E}$} \\
\hline Industrie I & $\mathrm{L}$ & $\mathrm{P}$ \\
\hline$+2,+2$ & $-3,+4$ \\
\hline$-4,-3$ & $-2,-2$ \\
\hline
\end{tabular}




\section{Le cas à trois industries}

Un autre cas de contagion protectionniste a lieu quand l'industrie des importables recherche des barrières à l'entrée des produits étrangers et quand l'industrie des exportables s'efforce d'obtenir des subventions à l'exportation. Cette politique conjuguée de droits de douane et de subventions à l'exportation est équivalente à une politique de dévaluation. Aussi, le modèle à deux industries n'est-il plus le mieux adapté pour examiner ce problème : il convient d'introduire une industrie des biens non échangeables, et les décideurs publics qui lui sont attachés, comme troisième joueur. Nous supposerons que cette industrie des biens non échangeables ne fait aucun usage d'importations dans ses processus de fabrication.

La question qui se pose alors est la suivante : un jeu non coopératif du type du dilemme du prisonnier est-il encore possible? La réponse est positive, et, comme l'a montré Rapoport [1971], le dilemme est encore plus sévère dans la version à trois joueurs que dans celle à deux. Le tableau 4 montre un exemple de dilemme du prisonnier à trois joueurs, construit dans sa forme extensive, où I représente l'industrie des importables (et les décideurs publics qui lui sont associés); $\mathbf{E}$ l'industrie des exportables et $\mathbf{N}$ celle des biens non échangeables. Le choix est, à nouveau, entre la stratégie de libre-échange (L) et celle de protection $(\mathrm{P})$. Les revenus sont donnés sous l'hypothèse que le niveau initial de protection existant dans le pays est tel que le choix du libre-échạnge, par l'ensemble des industries et des décideurs publics, assure un gain positif poúr l'ensemble de l'économie et pour chacun des joueurs, une fois les compensations faites. Cependant, si un seul, deux ou les trois joueurs s'efforcent de bénéficier de davantage de protection, les industries continuant à accepter la stratégie libre-échangiste enregistreront une perte, comme le veut la théorie usuelle de la protection.

Plus précisément, comme le souligne Rapoport, ce cas du dilemme du prisonnier peut se comprendre en termes de gains reçus par le joueur qui joue contre la «ationalité collective ». Si une industrie choisit la stratégie protectionniste, elle obtiendra le revenu le plus élevé $(+3)$. Si deux industries choisissent cette stratégie, elles obtiendront un revenu positif, mais plus faible $(+1)$. En l'absence de toute coalition, la pire solution, c'est-à-dire la contagion protectionniste, sera choisie par les trois industries : l'industrie des importables demandera des droits de douane ou des mesures équivalentes, l'industrie des exportables exigera des subventions à l'exportation, et enfin l'industrie 
des biens non échangeables cherchera à obtenir des mesures propres à augmenter le prix des biens non échangeables relativement à ceux des biens échangeables. A l'évidence, certaines de ces mesures seront en contradiction avec d'autres : par exemple, tel sera le cas entre les droits de douane qui augmentent le prix des importations (échangeables) par rapport aux prix des non échangeables, et les mesures demandées par l'industrie des biens non échangeables qui auront un effet inverse et diminueront le taux de change réel. Du fait de cet aspect contradictoire des mesures mises en cuvre, il est à nouveau possible que, selon la force relative des différentes mesures prises, le pays puisse éventuellement se retrouver avec des termes de l'échange domestiques qui se soient rapprochés des termes de l'échange internationaux à la fin du processus de contagion protectionniste. Mais il doit être rappelé que cette évolution vers une allocation des ressources plus compatible avec les prix relatifs internationaux s'est accompagnée d'un énorme processus de recherche de rentes, impliquant industries et décideurs publics, et donc contractant d'autant les dotations factorielles disponibles pour la production des biens et services destinés à la consommation finale.

Tableau 4. Contagion protectionniste avec trois industries

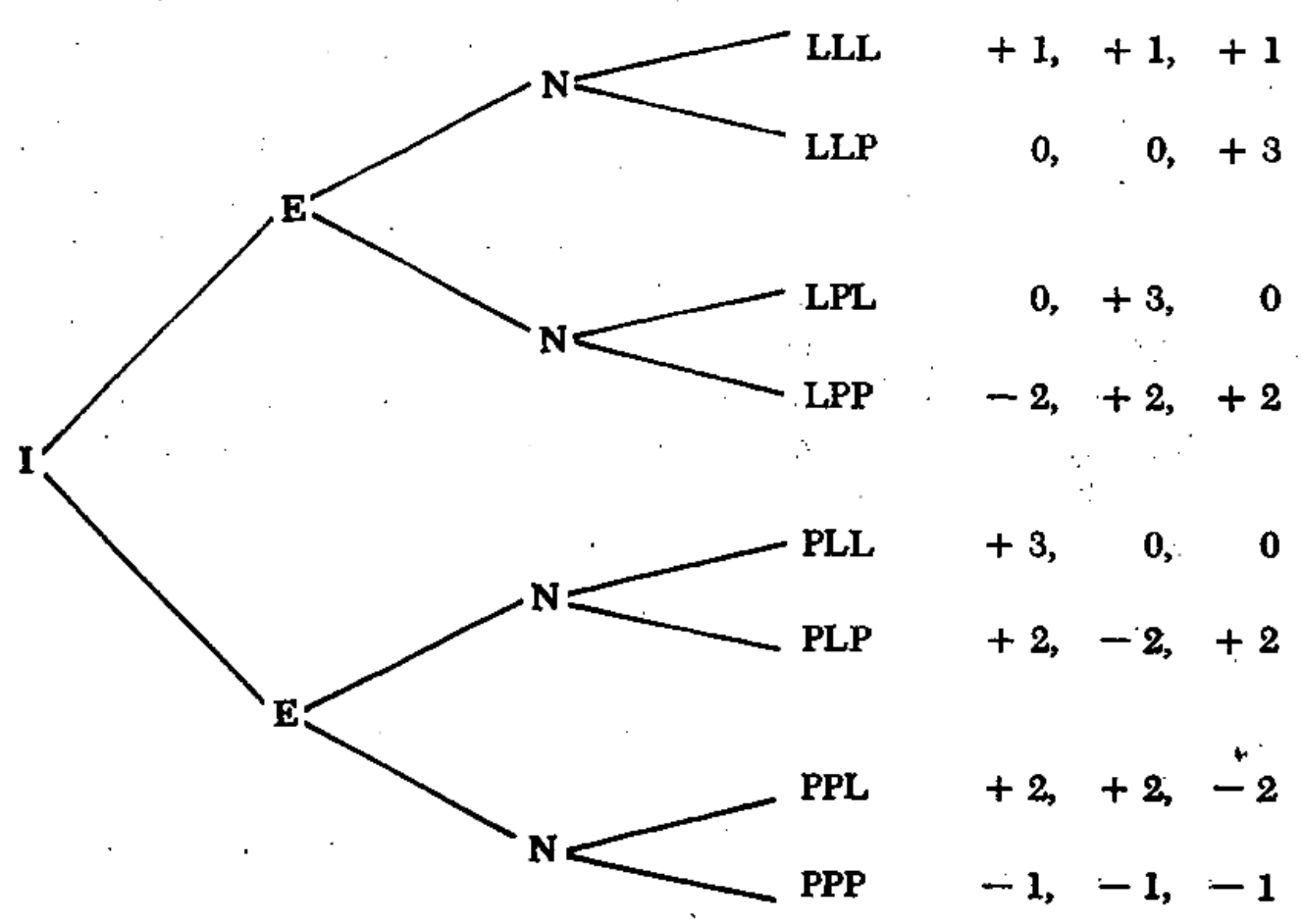




\section{Revue économique}

Pour conclure, il convient d'envisager la possibilité de coalitions. Comme nous avons supposé l'absence de toutes relations entre les industries des biens non échangeables et celle des biens échangeables (notamment en excluant le cas de biens intermédiaires importés et utilisés dans la production des biens non échangeables), toutes les coalitions sont envisageables sans qu'aucune puisse être considérée de façon privilégiée. Une caractéristique intéressante du jeu illustré par le tableau 4 est précisément qu’aucun joueur n'est intéressé à constituer une coalition avec un autre joueur. C'est cette caractéristique qui justifie l'affirmation de Rapoport selon laquelle la version du jeu non coopératif à trois joueurs est plus sévère que celle du dilemme à deux joueurs. Pour le problème qui nous intéresse, ceci renforce la vraisemblance de la contagion protectionniste.

\section{CONCLUSION}

La pénétration du marché intérieur est souvent présentée comme une des causes essentielles des forces protectionnistes. Cette assertion est cohérente avec les tests présentés dans ce papier. Deux autres forces, cependant, semblent jouer un rôle déterminant : les barrières non tarifaires tendent à protéger le facteur travail, qualifié ou non, alors que les droits de douane apparaissent liés à la structure industrielle de la branche, et tout particulièrement au niveau de concentration. Cette étude des structures de protection de l'économie française doit servir à éclaircir l'action des autorités publiques envers l'industrie. Il est vraisemblable que toute action insistant sur la concentration au sein des branches industrielles, par exemple à travers la réorganisation du secteur public, et que toute action en faveur du développement de la recherche-développement contiennent en ellesmêmes de fortes tendances à la protection. Céder à ces tendances reviendrait à accroître le coût, pour les consommateurs français, de ces politiques et à en diminuer les éventuels impacts favorables qui pourraient naître d'une vigoureuse compétition avec les économies étrangères.

\section{Patrick A. MESSERLIN}

Professeur de Sciences économiques Université de Lille I

et Service d'étude de l'activité économique (Fondation nationale des sciences politiques) 


\section{ANNEXE}

\section{Variables endogènes}

Droits de douane moyens : recettes douanières hors CEE rapportées aux importations hors CEE en NAP 40.

Droits de douane simples : moyenne arithmétique en NAP 40 des droits de douane moyens calculés en NAP 100 (hors CEE).

Droits de douane effectifs : calculés selon la formule :

$$
\mathrm{E}_{j}=\left\{\left(1-\sum_{i} a_{i j}\right) /\left(1 / 1+\mathrm{T}_{j}\right)-\left(\sum_{i} a_{i j} /\left(1+\mathrm{T}_{i}\right)\right)\right\}-1
$$

Barrières non tarifaires (valeur absolue) : nombre d'obstacles non tarifaires constatés pondérés par les importations.

Barrières non tarifaires (en pourcentage des importations).

\section{Variables exogènes}

CNQ : Part de marché des quatre premières firmes.

SIQ : Nombre de travailleurs qualifiés sur l'emploi total.

RDQ : Nombre d'ingénieurs et techniciens assimilés sur l'emploi total.

SAQ : Estimation du coefficient de l'équation de Hufbauer : VA $=a L_{i}^{\alpha}$.

LSH : Rémunération salariale (R 10) sur valeur ajoutée.

KLQ* : Capital par employé. * Les estimations du stock du capital utilisées sont celles du modèle PROPAGE.

DIM : Variation relative des importations (1970-1974).

CIQ : Coefficient de Grubel et Lloyd.

VSH : Valeur ajoutée sur production totale.

AWQ : Rémunération moyenne par salarié.

ESQ : Exportations sur production domestique.

ISQ : Importations sur consommation apparente.

DTC : Variation (1970-1974) de la consommation intermédiaire de transports rapportée à la production. 


\section{BIBLIOGRAPHIE}

[1979] ANDERson K., Politico-economic factors affecting structural change and adjustment $"$ in The economics of structural change and adjustment, ed. par C. Aislabie et C. Tisdell, Institute of Industrial Economics Conférence, séries 5, Université de Newcastle, Australie.

[1981] Anderson K., BaLdwin R.E.; * The political market for protection in Industrial countries : empirical evidence ", World Bank Staff Working Paper, 492.

[1982] Bar.assa B., "Les tendances actuelles de la spécialisation internationale de la production manufacturière $n$ in Internationalisation et autonomie de décision : les choix français, H. Bourguinat, ed., Paris, Economica.

[1978] Bairoch P., a Protectionnisme et expansion en Europe de 1892 à 1914 », Relations Internationales.

[1982] BaLdwin R.E., U.S. political pressures against adjustment to greater imports" in Trade and growth in the advanced developing countries, ed. par W. Hong et L. Krause, Seoul et Honolulu, KDI Press et Université Hawaii Press.

[1980] BoBE B., "The determinants for protection : The french case n, Working Paper.

[1974] BRETON A., The economic theory of representative government, Chicago, Aldine.

[1976] Caves R. E., "Economic models of political choice : Canada's tariff structure n, Canadian Journal of Economics, 9 (2), 278-300, mai.

[1981] Caves R.E., Jones R. W., Economie internationale, Paris, A. Colin.

[1957] Downs A., An economic theory of democracy, New York, Harper and Row.

[1970] Johnson H.G., " A new view of the infant industry argument ", in Studies in international economics, Mc Dougall I. A., SNape R. H., ed., North Holland Pub. Cy.

[1971] Jones R. W., " A three-factor model in theory, trade and history $n$, in Trade balance of payments and growth, Bhagwati et al., ed. North Holland Pub. Cy.

[1974] Krugcer A. O., "The political economy of the rent secking society ", American Economic Review, juin.

[1980] Krugman P., "Scale economies, product differenciation, and the pattern of trade ", American Economic Review, décembre.

[1979] Magee S.P., Brocx W. A., " The economics. of special-interest politics : the case of the tariff ", American Economic Review, 68 (2), 246-250, mai.

[1980] Messerlin P. A., "Croissance et politiques commerciales : quelques interrogations sur le cas français (1830-1914) ». A paraître dans les Mélanges en I'honneur de J. Weiller, Paris, Economica.

[1982] Messeri.in P.A., Estimations de la protection effective en France en NAP 40 et en NAP 100, SEAE, miméo.

[1965] Orson M.; The logic of collective action, Cambridge, Harvard University Press. 


\section{Bibliographie}

[1975] Pincus J. J., A positive theory of tariff formation applied to the 19th century united states, publié par Ph. D. Dissertation, Université de Stanford.

[1957] Prouteau R., Recherches sur la loi des proportions de facteurs en commerce international, Thèse ès sciences économiques, Université de Poitiers.

[1971] Rapoport A., N-Person game theory, Université de Michigan Press.

[1981] RAY E: J., " Determinants of tariff and nontariff trade restrictions in the United States ", Journal of Political Economy, février.

[1974] Sénat des Etats-Unis, Hearings, US Tariff Commission, Report on the obstacles to trade.

[1978] Vellas F., Facteur travail et commerce international, thèse ès sciences économiques, Université de Toulouse. 